\title{
Composición y diversidad florística de bosques secos en la Meseta de Cacaxtla, Sinaloa, México
}

\author{
Composition and floristic diversity of dry forests in the Meseta de Cacaxtla, Sinaloa, Mexico
}

\section{Gilberto Márquez-Salazar ${ }^{1}$, Bladimir Salomón-Montijo1*, Álvaro Reyes-Olivas ${ }^{2}$, Marisol Amador-Medina ${ }^{3}$ \& Guillermo Millán-Otero ${ }^{4}$}

${ }^{1}$ Facultad de Biología, Universidad Autónoma de Sinaloa, Culiacán, Sinaloa, México.

${ }^{2}$ Colegio de Ciencias Agropecuarias, Facultad de Agronomía del Valle del Fuerte, Universidad Autónoma de Sinaloa, Juan José Ríos, Ahome, Sinaloa, México.

${ }^{3}$ Comisión Nacional de Áreas Naturales Protegidas, Mazatlán Sinaloa, México.

${ }^{4}$ Centro de Investigación en Alimentos y Desarrollo, Mazatlán, Sinaloa, México.

*Email: vladimir.salomon@uas.edu.mx

\section{RESUMEN}

Se estudió la composición florística, riqueza $(S)$, heterogeneidad $\left(H^{\prime}\right)$, equidad $\left(J^{\prime}\right)$ y similitud en y entre el bosque tropical caducifolio (BTC) y bosque espinoso (BE) de cuatro sitios del Área de Protección de Flora y Fauna Meseta de Cacaxtla, Sinaloa, México de junio a noviembre de 2016. En el área se localizan los parches más meridionales del bosque espinoso en el noroeste de México, comparándose por primera vez en la región ambos bosques secos. Se realizaron transectos lineales de $50 \times 2 \mathrm{~m}$ replicados 10 veces, sumando 0,1 ha, ordenando por familias, géneros, especies, formas de crecimiento cada uno de los individuos registrados y se obtuvieron valores por los coeficientes de Shannon-Wiener, Pielou y Jaccard. Resultaron 28 familias, 68 géneros y 92 especies de formas arbóreas, arbustivas y trepadoras. Las familias con mayor riqueza de géneros, especies y formas de crecimiento fueron Fabaceae, Cactaceae, Euphorbiaceae y Malvaceae; los géneros más diversos en especies fueron: Bursera, Caesalpinia y Randia. El BTC registró 83 especies y el BE 69. Los valores de heterogeneidad $H^{\prime}$ fueron altos, y variaron de 3,20 hasta 3,65 bits/ind. Los valores de equidad $\left(J^{\prime}\right)$ oscilaron entre 0,74 y 0,86. La similitud, arrojó valores entre 0,338 y 0,479. La comparación entre Las Labradas (BE) y El Rancho Las Palomas resultó en una menor cantidad de especies compartidas, presentando la mayor tasa de recambio de especies entre ambos bosques secos. La composición y diversidad florística fue alta en los bosques secos representativos de la Meseta de Cacaxtla.

Palabras clave: bosque espinoso, bosque tropical caducifolio, diversidad alfa, equidad, similitud.

\section{ABSTRACT}

The floristic composition, richness $(S)$, heterogeneity $\left(H^{\prime}\right)$, evenness $\left(J^{\prime}\right)$ and similarity were studied inside and between the tropical deciduous forest (BTC) and thorn scrub (BE) of four sites from the Area of Protection of Flora and Fauna Meseta de Cacaxtla, Sinaloa, Mexico from June to November 2016. In this area are located the southernmost patches of thorn scrub in northwest Mexico, comparing for the first time in the region both dry forests. Linear transects measured $50 \times 2$ meters were replicated 10 times adding to 0.1 ha, then each of the registered individuals were ordered by families, genera, species and growth forms and at last the values were obtained by the Shannon-Wiener, Pielou and Jaccard coefficients. There were found 28 families, 68 genera and 92 species of arboreal, shrub and climbing forms. The families with higher richness of genera, species and forms of growth were Fabaceae, Cactaceae, Euphorbiaceae and Malvaceae; the most diverse genera in species were: Bursera, Caesalpinia and Randia. The BTC registered 83 species and the BE 69. The heterogeneity values $\left(H^{\prime}\right)$ 
were high, and varied from 3.20 to 3.65 bits/ind. The evenness values $\left(J^{\prime}\right)$ ranged between 0.74 and 0.86 . The similarity generate values between 0.338 and 0.479 . The comparison between Las Labradas (BE) and El Rancho Las Palomas resulted in a lower number of shared species, presenting the highest rate of species turnover between both dry forests. The floristic composition and diversity were high in the representative dry forests of the Meseta de Cacaxtla.

Keywords: alpha diversity, evenness, similarity, thorn scrub, tropical deciduous forest.

\section{INTRODUCCIÓN}

La Meseta de Cacaxtla fue decretada como Área de Protección de Flora y Fauna (APFFMC) en el año 2000 (DOF 2000). El sitio presenta una notoria heterogeneidad de ambientes, es reservorio de especies endémicas, corredor biológico y brinda servicios ecológicos; se caracteriza por una amplia representatividad de ecosistemas (SEMARNAT 2015) y una diversidad de comunidades vegetales de bosques secos (bosque tropical caducifolio y bosque espinoso), semisecos (bosque tropical subcaducifolio) y húmedos (vegetación riparia y manglares). Los bosques secos, bosques tropicales secos o bosques tropicales estacionales secos (Murphy \& Lugo 1995) incluyen a los bosques cuyos árboles y arbustos se les caen total o parcialmente sus hojas en la época de ausencia de precipitaciones o de sequía, que se prolonga cinco o más meses al año (Maass \& Burgos 2011). La marcada estacionalidad afecta la composición de especies, estructura y funcionamiento de estos bosques (Fajardo et al. 2005). En México se localizan separados en ambas vertientes geográficas, por el Pacífico se distribuyen desde el sur de Sonora y Baja California Sur hasta Chiapas (Rzedowski 1978); su disyunción facilita un alto grado de endemismo y de especiación. Posee una riqueza biológica única, con especies pertenecientes principalmente a las familias Fabaceae, Bignoniaceae, Malvaceae, Apocynaceae, Capparaceae, Euphorbiaceae, Cactaceae, Rubiaceae, Burseraceae, Moraceae (Murphy \& Lugo 1986; Gentry 2009; Álvarez-Yépiz et al. 2008; Apgaua et al. 2015; Bravo et al. 2016); predominan las formas de crecimiento arbóreas sobre las lianas (Méndez-Toribio et al. 2014). Se caracteriza por una heterogeneidad florística entre parches de vegetación, así como bajos valores de diversidad (Gentry 1988; Pennington et al. 2009) puntual, con altas tasas de recambio florístico (Trejo \& Dirzo 2002) que se incrementan con la distancia y entre sitios (Balvanera et al. 2002). Los bosques secos, a pesar de la elevada riqueza taxonómica, heterogeneidad ecológicafuncional y complejidad estructural, son los ecosistemas tropicales más amenazados del mundo (Janzen 1988), con alrededor del $60 \%$ de su superficie actual extinta, quedando bosques que experimentan altos niveles de fragmentación (Sánchez-Azofeifa et al. 2014). En el noroeste de México los bosques secos de la planicie costera noroccidental de Sonora y Sinaloa se fragmentaron desde la década de 1940 a 1950, para construir grandes obras de irrigación, que cambiaron el uso del suelo de grandes extensiones de bosques espinosos y matorrales xerófilos a parcelas agrícolas y ganaderas (Rzedowski 1978, Rohwera et al. 2010, Maass et al. 2010). En décadas recientes la pérdida continúa, destinándose superficies de comunidades vegetales costeras al cultivo de camarón, reduciendo la superficie a fragmentos inconexos, rodeados de campos de cultivo y pastizales (Sicairos et al. 2003). Debido a la problemática es urgente conocer cómo se encuentran estos bosques; para tal propósito se plantearon los siguientes objetivos: 1. Determinar la composición florística, 2. Cuantificar la diversidad alfa (a) y similitud en y entre bosques secos del Área de Protección de Flora y Fauna Meseta de Cacaxtla, Sinaloa, México.

\section{MATERIALES Y MÉTODOS}

\section{ÁREA DE ESTUDIO}

El estudio se realizó en el Área de Protección de Flora y Fauna Meseta de Cacaxtla, situada en la parte costera de los municipios de San Ignacio y Mazatlán, en la región centro sur de Sinaloa $\left(106^{\circ} 35^{\prime} 10^{\prime \prime}\right.$ y $106^{\circ} 45^{\prime} 30^{\prime \prime}$ oeste; $23^{\circ} 32^{\prime}$ $00^{\prime \prime}$ y $23^{\circ} 46^{\prime \prime} 00^{\prime \prime}$ norte), con una extensión de 50, 862-3125 ha (Figura 1). Posee un gradiente de elevación de los 0 a los $360 \mathrm{msnm}$. El clima es seco o estepario BS1(h') w (w), la temperatura promedio anual es de $23,2^{\circ} \mathrm{C}$ y una precipitación media anual de $577,3 \mathrm{~mm}$, con el $80 \%$ de esta entre julio y octubre (García 2004). Los tipos de suelo dominantes son Litosol, Regosol y Vertisol según la clasificación FAO/ UNESCO (INEGI 1988).

La vegetación dominante es bosque tropical caducifolio (BTC), bosque espinoso (BE) y parches de bosque tropical subcaducifolio (BTS), principalmente a orilla de arroyos (Rzedowski 1978). 


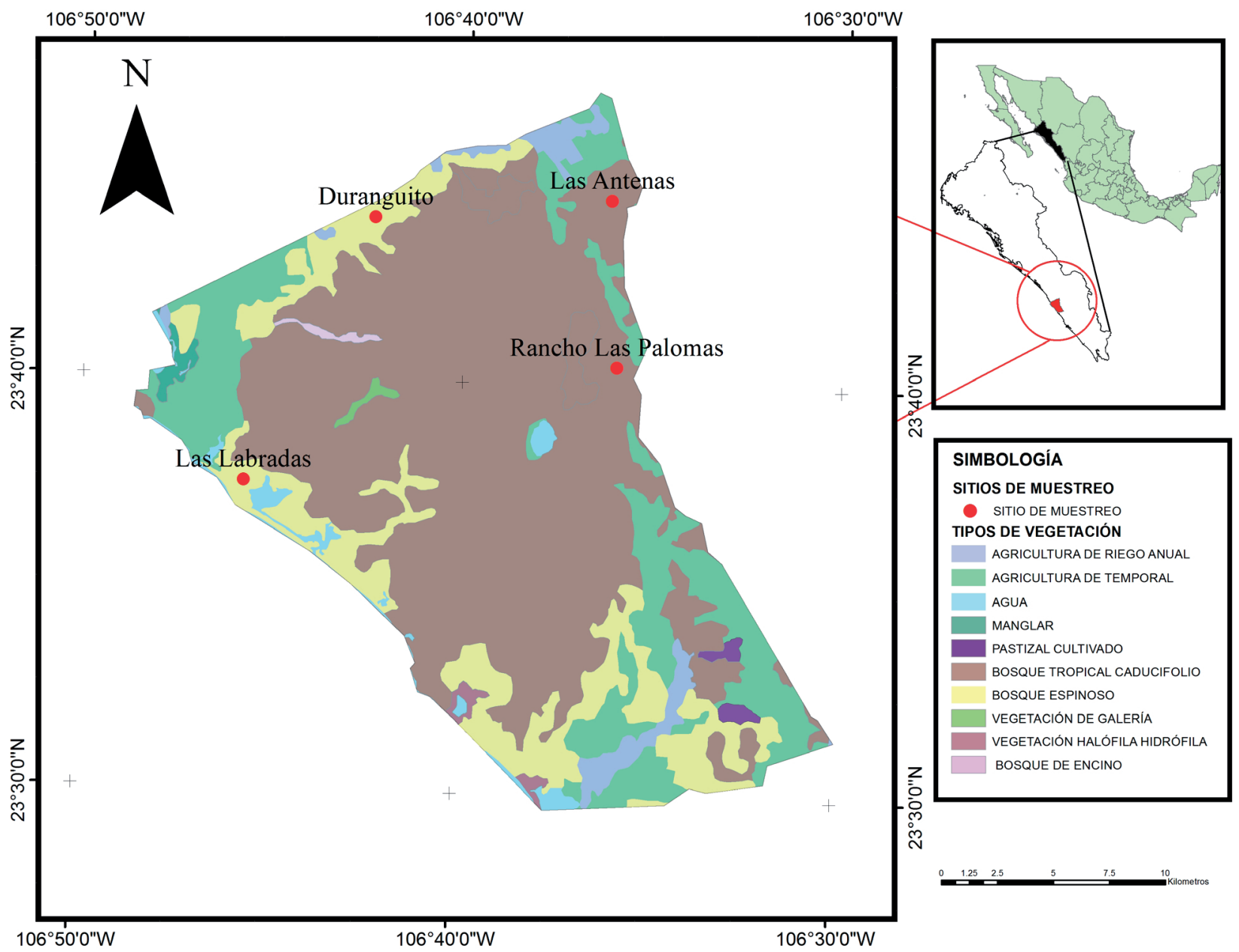

Figura 1. Localización del área de estudio, Área de Protección de Flora y Fauna Meseta de Cacaxtla, Sur de Sinaloa, México. / Location of area of study, Flora and Fauna Protection Area Meseta de Cacaxtla.

\section{Sitios de muestreo}

Sobre el polígono con los tipos de vegetación de bosque tropical caducifolio (BTC) y bosque espinoso (BE) (Rzedowski 1978), se trazó una malla reticulada escala 1:250 000, en la cual se seleccionaron de manera aleatoria estratificada cuatro sitios de muestreo, dos por cada comunidad vegetal; las unidades de medición fueron transectos con dimensiones de $2 \times 50$ m (Gentry 1982, 1988), replicados 10 veces, adyacentes entre sí y perpendiculares a la pendiente, que sumaron por sitio $1000 \mathrm{~m}^{2}$ (0,1 ha), estableciendo 40 transectos en una superficie de $4000 \mathrm{~m}^{2}$. Los sitios designados por las localidades o predios donde se ubicaron fueron: Las Labradas y Duranguito con BE y Rancho Las Palomas y Las Antenas con BTC (Figura 1). Se registraron individuos pertenecientes a especies arbóreas con un grosor de DAP $\geq 5 \mathrm{~cm}$, a una altura de $1.30 \mathrm{~m}$ (Williams-Linera \& Lorea 2009; López-Martínez et al. 2013; Dias et al. 2017); para formas arbustivas y trepadoras $\geq 2,5 \mathrm{~cm}$ (Gentry 1988). Los muestreos se realizaron de junio a noviembre del 2016.

Identificación de plantas. Se recolectaron los especímenes y utilizaron las técnicas convencionales para su herborización (Díaz 2007); para la nomenclatura de familias, géneros y especies se consultó Tropicos, en el Botanical Information System at the Missouri Botanical Garden (www.tropicos.org); se apoyo en taxónomos de las Unidades Académicas Facultad de Biología de la Universidad Autónoma de Sinaloa y el Jardín Botánico Culiacán; se revisó bibliografía especializada (Smith 
et al. 2004, Iltis \& Cornejo 2010, Hernández \& Vega 1989, Vega \& Villaseñor-Ríos 2008).

Diversidad alfa (a). Se contó la riqueza $(S)$, o número de especies (Halffter \& Moreno 2007) de los sitios muestreados y se cuantificó la heterogeneidad $\left(H^{\prime}\right)$; se empleó el índice de Shannon-Wiener, el cual está basado en la teoría de la información (Krebs 1999) y ha sido utilizado comúnmente para medir diversidad de especies, comprende riqueza de especies, y uniformidad o equidad (Ludwig \& Reynolds 1988). La función de Shannon-Wiener es:

$$
H^{\prime}=-\sum_{i=1}^{s} P_{i} \log _{2} P_{i}
$$

Donde $P_{i}=$ es el valor de la abundancia relativa de la i-ésima especie respecto al total de la muestra; $\log _{2=}$ logaritmo base dos. Magurran (2004) mencionó que 3.5 bit/ind era el valor más alto de $H^{\prime}$ por el coeficiente de Shannon-Wiener y que raramente superaba 4 . Los valores altos indican comunidades más uniformes donde la distribución de la abundancia es más homogénea y la dominancia de algunas especies es menor y contrariamente es baja en comunidades transitorias, explotadas, con condiciones ambientales fluctuantes (Margalef 1982), sujetas a perturbaciones estacionales o periódicas, ya sea por parte del hombre o la naturaleza (Odum 1972).

Para obtener la uniformidad o equidad $\left(J^{\prime}\right)$ se empleó la función de Pielou (1975):

$$
J^{\prime}=\frac{H^{\prime}}{\log _{2} S}
$$

Donde $H^{\prime}$ es la heterogeneidad, $\log _{2}$ es el logaritmo base dos, y $S$ es el número de especies; valores bajos de equidad indican predominancia de una o algunas especies sobre el resto de ellas. Ambos índices se calcularon con el software EcoStat (Towned 1999), luego se compararon entre y dentro de comunidades con la prueba HSD (honestly significant difference) de Tukey al 5\%, después de corroborar la normalidad de los datos; este análisis se realizó con el programa estadístico SAS (SAS Institute Inc. 2014).

Similitud en y entre comunidades. Para determinar las semejanzas entre sitios o muestras de las comunidades, se empleó el coeficiente de Jaccard (Magurran 1988):

$$
C_{j}=\frac{j}{(a+b-j)}
$$

Donde $j$ son las presencias compartidas, $a$ son las especies exclusivas del sitio 1 y $b$ las especies exclusivas del sitio 2 . Los resultados del índice tienen una escala del cero al uno. El cero indica muestras o comunidades completamente diferentes, no existe ensamblaje; por el contrario el uno indica qué son iguales (Magurran 1988) y el ensamblaje es total.

El coeficiente de similitud se determinó dentro y entre sitios del bosque tropical caducifolio y bosque espinoso, para lo cual se empleó el programa EcoStat (Towned 1999).

\section{RESULTADOS}

\section{COMPOSICIÓN FLORÍSTICA}

Se identificaron 28 familias, 68 géneros y 92 especies de formas arbóreas, arbustivas y trepadoras en cuatro sitios de estudio en bosques secos del APFFMC. Los géneros más variables en especies fueron: Bursera con 6, Caesalpinia 5, Randia 4; Euphorbia, Jatropha, Opuntia y Stenocereus 3 cada una, acumulando $29,35 \%$ de especies (Tabla 1 ).

Siete familias concentraron la mayor parte de géneros (40/68) y de especies (62/92); el BTC y BE agruparon 51,47 y $42,65 \%$ de géneros, 59,78 y $48,91 \%$ de especies respectivamente (Tabla 2). De forma de vida (árbol: $\mathrm{Ar}$; arbusto: Ab; trepadora: $\mathrm{Tr}$ ) resultaron 50 arbóreas (54,35\%); 36 arbustivas $(39,13 \%)$ y 6 trepadoras $(6,5 \%)$ (92 especies, $100 \%)$; por comunidad ocurrieron 46 y 36 formas arbóreas; 31 y 30 formas arbustivas, 6 y 3 trepadoras en el BTC y BE, respectivamente. Resultando el BTC con mayor riqueza de especies y de formas de crecimiento que su similar BE.

Se registraron cuatro especies de formas arbóreas con categoría de riesgo en la NOM-059 (SEMARNAT 2010). Pitaya de Martínez (Stenocereus martinezii (J.G. Ortega) Buxb.) con categoría protección especial (Pr), endémica a Sinaloa, México; amapa (Handroanthus impetiginosus (Mart. ex DC.) Mattos), sinónimo de Tabebuia palmeri Rose, categorizada como amenazada (A), no endémica; guayacán (Guaiacum coulteri A. Gray) amenazado y endémico; especies registradas en ambas comunidades de bosques secos, y vainillo o bolillo (Albizia occidentalis Brandegee var. occidentalis), amenazada, no endémica, sólo registrado en el bosque espinoso.

\section{DIVERSIDAD}

\section{Diversidad alfa (a). Riqueza de especies (S)}

La riqueza específica (S) por sitio varió de 46 especies en Las Labradas, a 57 en El Rancho Las Palomas; Duranguito y Las Antenas registraron un número similar de especies con 53 y 52 , respectivamente. 
TABLA 1. Listado de especies y formas de vida de los bosques secos del APFF Meseta de Cacaxtla. / Species and life form list of dry forests from APPF Meseta de Cacaxtla.

\begin{tabular}{|c|c|c|c|c|}
\hline Familia & Nombre científico & $\begin{array}{l}\text { Forma de } \\
\text { vida }\end{array}$ & $\begin{array}{l}\text { Bosque tropical } \\
\text { caducifolio }\end{array}$ & $\begin{array}{l}\text { Bosque } \\
\text { espinoso }\end{array}$ \\
\hline Anacardiaceae & Spondias mombin L. & $A b$ & * & * \\
\hline \multirow[t]{2}{*}{ Apocynaceae } & Plumeria rubra L. & $\operatorname{Ar}$ & $*$ & * \\
\hline & Marsdenia edulis S. Watson & $\operatorname{Tr}$ & * & \\
\hline \multirow[t]{2}{*}{ Bignoniaceae } & Dolichandra unguis-cati (L.) L.G.Lohmann & $\operatorname{Tr}$ & * & \\
\hline & Handroanthus impetiginosus (Mart. ex DC.) Mattos & $\operatorname{Ar}$ & * & * \\
\hline Boraginaceae & Cordia sonorae N.E.Rose & $\operatorname{Ar}$ & * & \\
\hline \multirow[t]{6}{*}{ Burseraceae } & Bursera excelsa (Kunth) Engl. & $\mathrm{Ar}$ & & * \\
\hline & Bursera fagaroides ((Kunth) Engl. & $\operatorname{Ar}$ & * & \\
\hline & Bursera grandifolia (Schltdl.) Engl. & $\mathrm{Ar}$ & * & * \\
\hline & Bursera laxiflora S. Watson. & $\mathrm{Ar}$ & * & * \\
\hline & Bursera penicillata (Sesse \& Mociño ex DC.) Engl. & $\operatorname{Ar}$ & * & * \\
\hline & Bursera simaruba (L.) Sarg. & $\operatorname{Ar}$ & * & * \\
\hline \multirow[t]{11}{*}{ Cactaceae } & Acanthocereus tetragonus (L.) Hummelinck & $A b$ & $*$ & * \\
\hline & Cylindropuntia thurberi (Engelm.) F.M.Knuth & $A b$ & * & * \\
\hline & Opuntia decumbens Salm-Dyck & $A b$ & * & * \\
\hline & Opuntia karwinskiana Salm-Dyck & $A b$ & * & * \\
\hline & Opuntia sp. & $A b$ & * & \\
\hline & $\begin{array}{l}\text { Pachycereus pecten-aboriginum (Engelm. ex S.Watson) Britton \& } \\
\text { Rose }\end{array}$ & $\operatorname{Ar}$ & * & * \\
\hline & Pereskiopsis porteri (Brandegee ex F.A.C.Weber) Britton \& Rose & $A b$ & * & * \\
\hline & Pilosocereus purpusii (Britton \& Rose) Byles \& G.D. Rowley & $A b$ & $*$ & $*$ \\
\hline & Stenocereus alamosensis (J.M. Coult) A.C. Gibson \& K.E. Horak & $A b$ & * & * \\
\hline & Stenocereus martinezii (J.G. Ortega) Buxb. & $\operatorname{Ar}$ & * & * \\
\hline & Stenocereus standleyi (J.G. Ortega) Buxb. & $A b$ & & * \\
\hline \multirow[t]{4}{*}{ Capparaceae } & Quadrella indica (L.) Iltis \& Cornejo & $\operatorname{Ar}$ & $*$ & $*$ \\
\hline & Crateva tapia L. & $\operatorname{Ar}$ & & * \\
\hline & Cynophalla flexuosa (L.) J.Presl & $A b$ & * & \\
\hline & Morisonia americana L. & $\mathrm{Ar}$ & & * \\
\hline Celastraceae & Pristimera celastroides (Kunth) A.C.Sm. & $A b$ & * & $*$ \\
\hline Combretaceae & Combretum farinosum Kunth & $\operatorname{Tr}$ & * & * \\
\hline \multirow[t]{2}{*}{ Convolvulaceae } & Ipomoea arborescens (Humb. \& Bonpl. ex Willd.) G. Don & $\operatorname{Ar}$ & * & * \\
\hline & Ipomoea bracteata Cav. & $\operatorname{Tr}$ & $*$ & * \\
\hline Ebenaceae & Diospyros aequoris Standl. & $\operatorname{Ar}$ & * & \\
\hline Erythroxylaceae & Erythroxylum mexicanum Kunth & $\mathrm{Ar}$ & $*$ & * \\
\hline Euphorbiaceae & Cnidoscolus sinaloensis Breckon ex Fern.Casas & $\mathrm{Ar}$ & $*$ & $*$ \\
\hline
\end{tabular}




\begin{tabular}{|c|c|c|c|c|}
\hline Familia & Nombre científico & $\begin{array}{c}\text { Forma de } \\
\text { vida }\end{array}$ & $\begin{array}{c}\text { Bosque tropical } \\
\text { caducifolio }\end{array}$ & $\begin{array}{c}\text { Bosque } \\
\text { espinoso }\end{array}$ \\
\hline & Croton alamosanus Rose & $\mathrm{Ab}$ & $*$ & * \\
\hline & Euphorbia californica Benth & $\mathrm{Ab}$ & $*$ & * \\
\hline & Euphorbia lomelii V.W.Steinm. & $A b$ & $*$ & \\
\hline & Euphorbia schlechtendalii Boiss. & $\operatorname{Ar}$ & * & \\
\hline & Jatropha cordata (Ortega) Müll.Arg. & $\mathrm{Ar}$ & $*$ & * \\
\hline & Jatropha curcas L. & $\operatorname{Ar}$ & $*$ & * \\
\hline & Jatropha aff. bullockii & $\mathrm{Ab}$ & * & * \\
\hline & Manihot rubricaulis I.M.Johnst. & $\mathrm{Ab}$ & $*$ & \\
\hline & Ricinus communis L. & $A b$ & $*$ & \\
\hline \multirow[t]{20}{*}{ Fabaceae } & Acacia cochliacantha Willd. & $A b$ & $*$ & * \\
\hline & Acacia farnesiana (L.) Willd. & $A b$ & * & * \\
\hline & Bauhinia pringlei S.Watson & $\mathrm{Ar}$ & $*$ & \\
\hline & Caesalpinia cacalaco Bonpl. & $\operatorname{Ar}$ & * & \\
\hline & Caesalpinia eriostachys Benth. & $\operatorname{Ar}$ & $*$ & * \\
\hline & Caesalpinia palmeri S. Watson & $\mathrm{Ab}$ & $*$ & * \\
\hline & Caesalpinia platyloba S. Watson & $\operatorname{Ar}$ & $*$ & * \\
\hline & Caesalpinia sclerocarpa Standl. & $\operatorname{Ar}$ & $*$ & * \\
\hline & Calliandra sp. & $A b$ & * & * \\
\hline & Chloroleucon mangense (Jacq.) Britton \& Rose & $\operatorname{Ar}$ & * & * \\
\hline & Conzattia sericea Stand. & $\operatorname{Ar}$ & $*$ & \\
\hline & Coursetia glandulosa A. Gray. & $\mathrm{Ab}$ & * & \\
\hline & Erythrina lanata Rose & $\operatorname{Ar}$ & $*$ & * \\
\hline & Haematoxylum brasiletto H. Karst. & $\operatorname{Ar}$ & * & * \\
\hline & Albizia occidentalis Brandegee & $\operatorname{Ar}$ & & * \\
\hline & Lonchocarpus guatemalensis Benth. & $\operatorname{Ar}$ & * & * \\
\hline & Lysiloma divaricatum (Jacq.) J.F. Macbr. & $\operatorname{Ar}$ & * & * \\
\hline & Pithecellobium dulce (Roxb.) Benth. & $\operatorname{Ar}$ & * & \\
\hline & Senna atomaria (L.) H. S. Irwin \& Barneby & $\mathrm{Ab}$ & * & * \\
\hline & Senna pallida (Vahl) H.S.Irwin \& Barneby & $\operatorname{Ar}$ & * & \\
\hline Malpighiaceae & Malpighia emarginata DC. & $\operatorname{Ar}$ & * & * \\
\hline \multirow[t]{5}{*}{ Malvaceae } & Byttneria aculeata Jacq. & $\operatorname{Tr}$ & * & \\
\hline & Ceiba aesculifolia (Kunth) Britten \& Baker f. & $\operatorname{Ar}$ & * & \\
\hline & Gossypium aridum (Rose \& Standl.) Skovst. & $\operatorname{Ar}$ & * & * \\
\hline & Guazuma ulmifolia Lam. & $\operatorname{Ar}$ & * & \\
\hline & Helicteres baruensis Jacq. & $\mathrm{Ab}$ & & * \\
\hline Moraceae & Maclura tinctoria (L.) D.Don ex Steud. & $\operatorname{Ar}$ & * & \\
\hline
\end{tabular}




\begin{tabular}{|c|c|c|c|c|}
\hline Familia & Nombre científico & $\begin{array}{c}\text { Forma de } \\
\text { vida }\end{array}$ & $\begin{array}{l}\text { Bosque tropical } \\
\text { caducifolio }\end{array}$ & $\begin{array}{c}\text { Bosque } \\
\text { espinoso }\end{array}$ \\
\hline & Ficus cotinifolia Kunth. & $\operatorname{Ar}$ & * & * \\
\hline Nyctaginaceae & Neea psychotrioides Donn.Sm. & $\operatorname{Ar}$ & * & * \\
\hline Passifloraceae & Turnera diffusa Willd. ex Schult. & $\mathrm{Ab}$ & & * \\
\hline Polygonaceae & Ruprechtia fusca Fernald & $\operatorname{Ar}$ & * & * \\
\hline Primulaceae & Bonellia macrocarpa (Cav.) B.Ståhl \& Källersjö & $\mathrm{Ab}$ & * & * \\
\hline \multirow[t]{3}{*}{ Rhammaceae } & Colubrina sp. & $A b$ & & * \\
\hline & Rhamnus humboldtiana Willd. ex Schult. & $\mathrm{Ab}$ & * & * \\
\hline & Ziziphus amole (Sessé \& Moc.) M.C. Johnst. & $\operatorname{Ar}$ & * & * \\
\hline \multirow[t]{6}{*}{ Rubiaceae } & Chiococca alba (L.) Hitchc. & $\mathrm{Ab}$ & & * \\
\hline & Hintonia latiflora (Sessé \& Moc. ex DC.) Bullock & $\mathrm{Ar}$ & * & \\
\hline & Randia armata (Sw.) DC. & $A b$ & * & * \\
\hline & Randia echinocarpa Moc. \& Sessé ex DC. & $A b$ & * & * \\
\hline & Randia obcordata S. Watson & $A b$ & * & * \\
\hline & Randia sp. & $A b$ & $*$ & * \\
\hline \multirow[t]{3}{*}{ Rutaceae } & Esenbeckia hartmanii B.L.Rob. \& Fernald & $\operatorname{Ar}$ & * & * \\
\hline & Zanthoxylum arborescens Rose & $\operatorname{Ar}$ & * & * \\
\hline & Zanthoxylum fagara (L.) Sarg. & $A b$ & * & * \\
\hline Salicaceae & Casearia arguta Kunth. & $\operatorname{Ar}$ & * & * \\
\hline \multirow[t]{2}{*}{ Sapindaceae } & Serjania sp. & $\operatorname{Tr}$ & * & * \\
\hline & Thouinidium decandrum (Humb. \& Bonpl.) Radlk. & $\operatorname{Ar}$ & * & \\
\hline Solanaceae & Lycium andersonii A. Gray & $A b$ & * & $*$ \\
\hline Zygophyllaceae & Guaiacum coulteri A. Gray & $\operatorname{Ar}$ & * & * \\
\hline
\end{tabular}

Nota: Forma de vida (árbol: Ar; arbusto: Ab; trepadora: Tr). / Life form (tree: Ar; shrub: Ab ; vine : Tr).

TABLA 2. Familias, géneros y especies dominantes en los bosques secos del APFFMC. / Dominant families, genus and species at the dry forests from APFFMC.

\begin{tabular}{lcccccc}
\hline Familia & \multicolumn{3}{c}{ Géneros } & & \multicolumn{3}{c}{ Especies } \\
& BTC & BE & Total & BTC & BE & Total \\
\hline Fabaceae & 13 & 10 & 14 & 19 & 14 & 20 \\
Cactaceae & 7 & 7 & 7 & 10 & 10 & 11 \\
Euphorbiaceae & 6 & 4 & 6 & 10 & 6 & 10 \\
Malvaceae & 4 & 2 & 5 & 4 & 2 & 5 \\
Capparaceae & 2 & 3 & 4 & 2 & 3 & 4 \\
Rubiaceae & 2 & 2 & 3 & 5 & 5 & 6 \\
Burseraceae & 1 & 1 & 1 & 5 & 5 & 6 \\
\hline$\Sigma$ & 35 & 29 & 40 & 55 & 45 & 62 \\
\hline
\end{tabular}




\section{Heterogeneidad $\left(\boldsymbol{H}^{\prime}\right)$}

Los valores de heterogeneidad por sitio de muestreo variaron de 3,20 bits/ind en Rancho Las Palomas, hasta los 3,65 bits/ind en Duranguito del bosque tropical caducifolio y bosque espinoso respectivamente. Las comparaciones con la prueba de Tukey HSD indican que este índice no difiere dentro y entre las comunidades de bosque espinoso y bosque tropical caducifolio $(P>0,05)$. La equidad difiere entre los sitios con BTC ( $P=0,01)$, con 0,86 en las Antenas y 0,74 en Las Palomas, pero no difieren respecto a los sitios de BE en Duranguito y Las Labradas, que tuvieron valores de 0,82 y 0,83 respectivamente (Tabla 3 ).

\section{Similitud}

Por el coeficiente de Jaccard los valores de similitud intra e inter comunidades variaron de 0,338a 0,479. La comparación entre Las Labradas y Rancho Las Palomas, con bosque espinoso y bosque tropical caducifolio respectivamente, donde se compartieron 28 especies, presentaron una baja similitud de 0,338; entre Las Antenas y Duranguito, con 35 especies en común, registraron una mediana semejanza de 0,479 (Tabla 4), existiendo una relación directa entre especies compartidas y similitud. Conforme más especies en común compartan las muestras, la similitud será mayor y los valores de parentesco resultantes serán más altos, contrastando con las muestras donde compartan menos especies en común, que por el contrario la similitud será menor y los valores resultaran más bajos. Contrariamente el valor bajo de similitud de 0,338 es un indicador de alta tasa de recambio de especies entre los sitios de ambos bosques secos.

TABLA 3. Índices de diversidad $\left(H^{\prime}\right)$ y equidad ( $\left.J^{\prime}\right)$ entre y dentro de tipos de vegetación. Los valores con igual superíndice no difieren estadísticamente (prueba HSD de Tukey, $P=0.05)$. / Diversity index $\left(H^{\prime}\right)$ and evenness $\left(J^{\prime}\right)$ among different types of vegetation. Values with the same superscript do not differ statistically (Tukey HSD test, $\mathrm{P}=0.05$ ).

\begin{tabular}{lcc}
\hline Sitios & $\begin{array}{c}\text { Shannon-Wiener } \\
\text { Heterogeneidad }\end{array}$ & $\begin{array}{c}\text { Pielou } \\
\text { Equidad }\end{array}$ \\
\hline Las Labradas (SBE) & $3,29^{\mathrm{a}}$ & $0,83^{\mathrm{ab}}$ \\
Duranguito (SBE) & $3,65^{\mathrm{a}}$ & $0,82^{\mathrm{ab}}$ \\
Rancho Las Palomas & $3,20^{\mathrm{a}}$ & $0,74^{\mathrm{a}}$ \\
Las Antenas & $3,52^{\mathrm{a}}$ & $0,86^{\mathrm{b}}$ \\
\hline
\end{tabular}

TABLA 4. Índices de similitud de Jaccard entre sitios. / Jaccard similarity index among sites.

\begin{tabular}{lcccc}
\hline Sitios & Las Labradas (BE) & Duranguito (BE) & $\begin{array}{c}\text { Rancho Las } \\
\text { Palomas (BTC) }\end{array}$ & $\begin{array}{c}\text { Las Antenas } \\
\text { (BTC) }\end{array}$ \\
\hline Las Labradas (SBE) & 1 & 0,394 & 0,338 & 0,343 \\
Duranguito (SBE) & & 1 & 0,467 & 0,479 \\
Rancho Las Palomas (SBC) & & & 1 & 0,473 \\
Las Antenas (SBC) & & & 1 \\
\hline
\end{tabular}

\section{DISCUSIÓN}

\section{COMPOSICIÓN FLORÍSTICA}

Las cantidades de familias, géneros y especies fueron variables por categoría taxonómica, comparada con algunos estudios de bosques secos; resultaron inferiores a las mencionadas por Bravo et al. (2016), quienes empleando un tamaño de muestra mayor, en Bahía de Banderas, Nayarit, México reportan 43 familias, 92 géneros y 127 especies; al igual que Powers et al. (2009), registraron en Costa Rica 45 familias y 135 especies; montos superiores a las citadas por Arriaga \& León (1989), quienes inventariaron bosques tropicales deciduos de Baja California Sur, en una superficie de muestreo similar, reportando 25 familias, 28 géneros y 67 especies; MéndezToribio et al. (2014), en un área estudiada inferior en la Depresión del Balsas, Michoacán, encontraron 24, 50 y 78 familias, géneros y especies, respectivamente, y similares a las reportadas por Trejo \& Dirzo (2002), para Cosalá, Sinaloa con 33 familias, 69 géneros y 80 especies. DRYFLOR (2016), reporta 1072 especies, cantidad obtenida de 33 sitios de 
bosques secos neotropicales de México, comparadas con las 92 especies descritas de cuatro sitios del APFF Meseta de Cacaxtla constituyen una pequeña cantidad del 8,58 \% del total registrado para estos bosques en México.

En relación a las familias con mayor riqueza de especies para los bosques secos neotropicales, los resultados coinciden en general con Pennington et al. (2009), para comunidades de América Latina y el Caribe, que mencionan a Leguminosae - Fabaceae, Capparaceae, Malvaceae, Cactaceae y Euphorbiaceae como las más conspicuas, y en particular para los bosque secos de México se concuerda con varios estudios que se han realizado (Arriaga \& León, 1989; Álvarez-Yépiz et al. 2008; Williams-Linera \& Lorea, 2009; Ávila Sánchez et al. 2010; Almazán-Núñez et al. 2012; Méndez-Toribio et al. 2014; Hernández-Ramírez \& García-Méndez, 2015; Bravo et al. 2016), en los que se menciona a Fabaceae, Euphorbiaceae, Burseraceae, Cactaceae, Rubiaceae y Malvaceae como las familias más importantes en este tipo de bosques. Con Lott \& Atkinson (2010), que describen la diversidad de especies de las selvas secas del occidente de México, se concuerda con Fabaceae, Euphorbiaceae y Cactaceae. Particularmente para el BTC de México se tiene similitud con Rzedowski \& Calderón (2013), en Fabaceae, Malvaceae, Cactaceae y Euphorbiaceae. Comparando la cantidad y porcentajes de géneros de las familias más ricas en especies del BTC de México con los del APFFMC, Euphorbiaceae registró seis géneros que constituyen 35,29\%, Cactaceae con siete alcanzó $29,17 \%$, Fabaceae con $13 \%$ obtuvo 15,29 \% y
Malvaceae con tres resultó con 9,68 \% (Tabla 5).

En relación a los géneros característicos de estos bosques, se coincidió con Bursera, el cual es el más común en diferentes estudios de composición florística de los bosque secos neotropicales del continente americano (Pennington et al. 2009), y para México (Méndez-Toribio et al. 2014; AlmazánNúñez et al. 2012; Rzedowski \& Calderón, 2013; WilliamsLinera \& Lorea, 2009), donde se ha reportado a Bursera, Caesalpinia, Randia, Euphorbia, Jatropha, Opuntia y Stenocereus entre los géneros más importantes del BTC. Son significativos además por su distribución restringida. Lott \& Atkinson (2010) mencionan a Bursera, Caesalpinia, Euphorbia y Jatropha como taxones especialmente notables en endemismos para las selvas secas del Pacífico de México.

Con respecto a las formas de vida en bosques secos del Pacífico de México, se repite el patrón de registro de mayor diversidad de formas arbustivas que de trepadoras; tal como lo encontrado en los bosques secos en Sonora, en el noroeste de México (Álvarez-Yépiz et al. 2008); en Guerrero, en el suroeste de México (Almazán-Núñez et al. 2012), y en la zona costera de Nayarit (Bravo et al. 2016).

Las especies arbóreas con categoría de riesgo en la NOM059 (SEMARNAT 2010) se caracterizaron por presentar frecuencia y abundancias heterogéneas en los sitios de muestreo. Handroanthus impetiginosus se registró en los cuatro lugares de estudio, sumó la mayor abundancia con 31 individuos y Albizia occidentalis la menor frecuencia con un sitio de registro y dos individuos (Tabla 6).

TABLA 5. Comparación de géneros por familia del BTC de México y APFF Meseta de Cacaxtla. / Comparison of genus by family from the tropical dry forest (BTC) of Mexico and APFF Meseta de Cacaxtla.

\begin{tabular}{lccc}
\hline Familia & BTC México & BTC Cacaxtla & $\%$ \\
\hline Fabaceae & 85 & 13 & 15,29 \\
Cactaceae & 24 & 7 & 29,17 \\
Euphorbiaceae & 17 & 6 & 35,29 \\
Malvaceae & 31 & 3 & 9,68 \\
\hline
\end{tabular}

TABLA 6. Especies en la NOM-059-SEMARNAT 2010 y abundancia en APFF Meseta de Cacaxtla. / Species listed at NOM-059SEMARNAT 2010 and abundance at APFF Meseta de Cacaxtla.

\begin{tabular}{lcccc}
\hline Sitio & $\begin{array}{c}\text { Handroanthus } \\
\text { impetiginosus }\end{array}$ & $\begin{array}{c}\text { Guaiacum } \\
\text { coulteri }\end{array}$ & $\begin{array}{c}\text { Stenocereus } \\
\text { martinezii }\end{array}$ & $\begin{array}{c}\text { Albizia } \\
\text { occidentalis }\end{array}$ \\
\hline Las Labradas & 3 & 0 & 0 & 0 \\
Duranguito & 1 & 5 & 3 & 2 \\
R. Las Palomas & 7 & 12 & 5 & 0 \\
Las Antenas & 20 & 0 & 3 & 0 \\
\hline$\Sigma$ & 31 & 17 & 11 & 2 \\
\hline
\end{tabular}


Los registros son similares a los de Rubio et al. (2010), quienes reportan amapa ( $H$. impetiginosus) para la Meseta de Cacaxtla, como especie amenazada (A). Pitaya de Martínez (S. Martinezii), guayacán (G. coulteri) y bolillo (A. occidentalis) no tenían reportes como especies en riesgo dentro del ANP.

\section{DIVERSIDAD}

Diversidad alfa (a). Riqueza de especies (S)

La riqueza varió entre 46 especies en Las Labradas, 52 en Las Antenas, 53 en Duranguito y 57 en el Rancho Las Palomas. Gentry (2009) mencionó que los bosques secos neotropicales tienen, en 0,1 ha, entre 50 y 70 especies, con un promedio de 64,9. Las especies registradas en los cuatro lugares de estudio están por debajo del promedio; Las Antenas, Duranguito y Rancho Las Palomas están por encima del límite inferior y Las Labradas está por debajo de las 50 especies. La riqueza registrada en los cuatro sitios de estudio del APFFMC se encuentra en los rangos reportados por diferentes autores para bosques secos tropicales; Lima \& Lima (1998) para la Catinga en Brasil inventariaron entre 39 y 71 especies; Gillespie et al. (2000) en Costa Rica y Nicaragua entre 45 y 75 especies; y Trejo \& Dirzo (2002) para 20 sitios con bosques secos en México entre 29 y 123 especies.

\section{Heterogeneidad $\left(H^{\prime}\right)$}

Los valores de $H^{\prime}$ fueron altos, en los cuatro sitios superaron los 3 bits/ind; al respecto Magurran (2004) mencionó que 3,5 era el valor más alto del índice de Shannon-Wiener y que raramente superaba 4. El Rancho Las Palomas y Las Labradas del BTC y BE registraron 3,20 y 3,29 bits/ind, respectivamente, con un rango de 3 y 3,5 bits/ind. Las Antenas y Duranguito del BTC y BE cuantificaron 3,52 y 3,65 bits/ind, respectivamente, los cuales superaron el valor alto de 3,5 bits/ind. Ningún bosque analizado en este estudio mostró un patrón claro de valores menores o mayores de $H^{\prime}$.

Los valores $H^{\prime}$ obtenidos fueron superiores, similares e inferiores a otros realizados en bosques secos tropicales, como los reportados por Alvarado et al. (2015), cuantificando la heterogeneidad en el Municipio Torres, Estado Lara, Venezuela donde se registraron valores bajos de 1,07 y 1,75; López-Toledo et al. (2012) en la reserva de la biósfera la Sepultura, Chiapas reportaron cantidades entre 0,92 y 2,98 ; Dzib-Castillo et al. (2014) en selvas secas y semi secas de Campeche encontraron entre 0,19 y 2,25 ; los valores fueron inferiores a 3 bits/ind; por su parte López et al. (2015), en Río Hato, Panamá obtuvieron 3,13; De Oliveira et al. (2015) en la Catinga al norte de Minas Gerais, Brasil entre 3,49, 3,61 y 3,74; Méndez-Toribio et al. (2014) en la Depresión del Balsas, Michoacán, México entre 3,41 y 3,43; Williams-
Linera \& Lorea (2009) en el Centro de Veracruz, México 3,76 , con valores superiores a 3 e inferiores a 4; y Riquetti et al. (2014) del norte de Minas Gerais, Brasil con un valor máximo de 4,52 y Trejo \& Dirzo (2002) en Caleta Michoacán 4,14; valores superiores a 4 bits/ind; estos últimos autores reportan para Cosalá, Sinaloa, un sitio cercano a los lugares de estudio, 3,81 de $H^{\prime}$ un valor alto cercano al registrado en Duranguito (3,65 bits/ind).

\section{EQUIDAD ( $\left.J^{\prime}\right)$}

Los resultados de equidad ( $\left.J^{\prime}\right)$ oscilaron entre 0,74 y 0,86 en Rancho Las Palomas y Las Antenas, respectivamente, ambos del BTC, los cuales resultaron superiores a los obtenidos por Dias et al. (2017) en el bioma Cerrado y zonas de transición del Cerrado y Catinga Brasil quienes registraron valores de 0,71 y 0,83; Riquetti et al. (2014), registraron valores oscilantes entre 0,619 y 0,850; De Oliveira et al. (2015) reportan 0,80, 0,84 y 0,85 ; similar y superior Méndez-Toribio et al. (2014) cuantificaron 0,86 y 0,89 .

Una marcada diferencia entre el número de individuos de la especie dominante y su contraparte codominante, arroja valores inferiores de equidad; por el contrario una menor disimilitud entre las densidades resulta en valores superiores de uniformidad. Una posible explicación del valor J' 0,74 del Rancho Las Palomas puede ser por la notoria oscilación en abundancia entre la especie dominante el arbusto vara blanca (Croton alamosanus), que registró una densidad promedio de 40,6 individuos, y las especies codominantes 9,1 individuos; la diferencia fue de 31,5 individuos; en el valor J' de 0,86 en Las Antenas, C. alamosanus cuantificó en promedio 15 y las codominantes 5,4 individuos; la oscilación fue de 9,6 individuos. Los valores de equidad $(J ')$ están influenciados por el arbusto vara blanca (C. alamosanus), abundante en sitios perturbados de bosques secos del APFFMC.

\section{SIMILITUD}

Los valores registrados en diferentes estudios de bosques secos resultan oscilantes entre bajos y medianos en similitud. De los estudios de Chamela, Jalisco México, Balvanera et al. (2002) mencionan un rango de 0,30 y 0,79; del noroeste de México Álvarez-Yépiz et al. (2008) entre 0,49 y 0,68; Williams-Linera \& Lorea (2009) de 0.13 y 0.62 , y de Tolima en el Valle del río Magdalena, Colombia, Fernández-Méndez et al. (2013) citan cantidades bajas de entre 0,02 a 0,23. Los valores bajos en similitud indican diferencias mayores y tasas altas de recambio en especies y por el contrario las cantidades altas de similitud arrojan menores diferencias en las muestras y tasas bajas de recambio en especies, entre sitios o comunidades. 


\section{CONCLUSIONES}

En el APFFMC convergen los bosques tropical caducifolio y bosque espinoso. En la composición de especies dominantes, de familias, géneros, formas de vida, estas fueron representativas de los bosques secos neotropicales. Fabaceae fue la más importante; Euphorbiaceae y Cactaceae registraron una alta cantidad de géneros característicos del BTC en México. Se registró a la mora (Maclura tinctoria), una especie ecológicamente generalista distribuida en los bosques secos del neotrópico (DRYFLOR 2016). Las especies en riesgo fueron de formas arboreas, no se registraron arbustos y trepadoras con protección especial, en peligro o amenazadas. La riqueza de especies (S) osciló en el rango inferior mencionado por Gentry (2009) para bosques secos neotropicales. Los valores de diversidad alfa $\left(H^{\prime}\right)$ fueron altos, superaron 3 bits/ind. La equidad $\left(J^{\prime}\right)$ fue alta, influenciada por la dominancia del arbusto vara blanca (Croton alamosanus). La similitud intra e inter sitios y comunidades no resultó en un patrón claro por bosque. Las mayores y menores similitudes no estuvieron relacionadas a las comunidades o la distancia entre sitios.

\section{AGRADECIMIENTOS}

A la Comisión Nacional de Áreas Naturales Protegidas por el financiamiento. A la Dirección General de Investigación y Posgrado de la Universidad Autónoma de Sinaloa. A Roberto Rodríguez, Jacek Márquez Stone, Juan Estrada Castelo y a Roberto Cárcamo Aréchiga, por la colaboración en el trabajo de campo y sugerencias al manuscrito.

\section{REFERENCIAS}

Almazán-Núñez, R. C., Del Coro, A. M., Eguiarte, L.E., Corcuera, P. 2012. Changes in composition, diversity and structure of woody plants in successional stages of tropical dry forest in southwest Mexico. Revista Mexicana de Biodiversidad 83: 1096-1109.

Alvarado, H., Rondón, I., Mondragón, A. 2015. Florística y estructura de dos espinares intervenidos en San Francisco, Municipio Torres, Estado Lara, Venezuela. Bioagro 27(3): 173-180.

Álvarez-Yépiz, J.C., Martínez-Yrízar, A., Búrquez, A., Lindquist, I. 2008. Variation in vegetation structure and soil properties related to land use history of old-growth and secondary tropical dry forests in northwestern Mexico. Forest Ecology and Management 256: 355-366.

Apgaua. D.M., Pereira, D.G.S., Santos, R.M., Menino, G.C.O.,
Pires, G.G., Fontes, M.A.L., TNG, D.Y.P. 2015. Floristic variation within seasonally dry tropical forests of the Caatinga Biogeographic Domain, Brazil, and its conservation implications. International Forestry Review 17(2): 33-44.

Arriaga, L., León, J.L. 1989. The Mexican tropical deciduous forest of Baja California Sur: a floristic and structural approach. Vegetatio 84: 45-52.

Ávila, S.P., Sánchez-González, A., Catalán, E.G. 2010. Estructura y Composición de la Vegetación del Cañón del Zopilote, Guerrero, México. Revista Chapingo Serie Ciencias Forestales y del Ambiente 16(2): 119-138.

Balvanera, P., Lott, E., Segura, G., Siebe, Ch., Islas, A. 2002. Patterns of $\beta$-diversity in a Mexican tropical dry forest. Journal of Vegetation Science 13: 145-158.

Bravo, B.O., Sánchez-González, A., De Nova, V.J.A., Pavón, H.N.P. 2016. Composición y estructura arbórea y arbustiva de la vegetación de la zona costera de Bahía de Banderas, Nayarit, México. Botanical Sciences 94(3): 603-623.

De Oliveira, M.G.C., Dos Santos, R.M., Apgaua, D.M.G., Gomes, P.G., Sales, P.D.G., Leite, F.M.A., De Souza, A. H. 2015. Florística e estrutura de florestas tropicais sazonalmente secas. CERNE 21(2): 277-291.

Diario Oficial de la Federación. 2000. Decreto por el cual se declara Área Natural Protegida, con el carácter de Área de Protección de Flora y Fauna, la región conocida como Meseta de Cacaxtla. México D.F. 8 pp.

Dias, G.A.P., Machado, E.L., Felfili, J.M., Rodrigues, P.J.R. 2017. Brazilian decidual tropical forest enclaves: floristic, structural and environmental variations. Revista brasileira de botanica 40(2): 417-426.

Díaz, P.W.A. 2007. Composición florística y estructura de bosques en los asentamientos campesinos Las Delicias, El Guamo y Lechozal, Estado Bolívar, Venezuela. ERNSTIA 17(1): 1-24.

Dryflor, 2016. Plant diversity patterns in neotropical dry forests and their conservation implications. Science 353(6306): 1383-1387.

Dzib-Castillo, B., Chanatásig-Vaca, C., González-Valdivia, N.A. 2014. Estructura y composición en dos comunidades arbóreas de la selva baja caducifolia y mediana subcaducifolia en Campeche, México. Revista Mexicana de Biodiversidad 85: 167-178.

Fajardo, L., González, V., Nassar, J.M., Lacabana, P., Portillo, G.C.A., Carrasquel, F., Rodríguez, J.P. 2005. Tropical Dry Forests of Venezuela: Characterization and Current Conservation Status. BIOTROPICA 37(4): 531-546.

Fernández-Méndez, F., Bernate-Peña, J.F., Melo, O. 2013. Diversidad arbórea y prioridades de conservación de 
los bosques secos tropicales del sur del departamento del Tolima en el Valle del río Magdalena, Colombia. Actualidades biológicas 35(99): 161-183.

García E. 2004. Modificaciones al Sistema de Clasificación Climática de Köppen. Instituto de Geografía. Universidad Nacional Autónoma de México. Serie de libros número 6. México D. F. 90 pp.

Gentry, A.H. 1982. Patterns of neotropical plant species diversity. Evolutionary Biology. 15: 1-54.

Gentry, A.H. 1988. Changes in plant community diversity and floristic composition on environmental and geographical gradients. Annals of the Missouri Botanical Garden 75: 1-34.

Gentry, A.H. 2009. Diversity and floristic composition of neotropical dry forests. In: Bullock, S.H., Mooney, H.A., Medina, E. (eds.), Seasonally dry tropical forests, pp. 146-194. Cambridge University Press, Cambridge, UK.

Gillespie, T.W., Grijalva, A., Farris, C.N. 2000. Diversity, composition, and structure of tropical dry forests in Central America. Plant Ecology 147: 37-47.

Halffter G., Moreno, C.E. 2007. Significado biológico de las diversidades Alfa, Beta y Gamma. En: Halffter, G., Soberón, J., Koleff, P., Melic, A. (eds.), Sobre diversidad biológica: El Significado de las diversidades alfa, beta y gamma, pp. 5-15 SEA, CONABIO, DIVERSITAS y CONACYT, México.

Hernández, A. F., Vega, R. 1989. Flora de la Península de Lucenilla. SEP y UAS. $24 \mathrm{pp}$.

Hernández-Ramírez, A.E., García-Méndez, S. 2015. Diversidad, estructura y regeneración de la selva tropical estacionalmente seca de la Península de Yucatán, México. Revista de Biología Tropical 63(3): 603-616.

Iltis, H., Cornejo, X. 2010. Studies in Capparaceae XXIX: synopsis in Quadrella, a Mesoamerican and West Indian genus. Journal of the Botanical Research Institute of Texas 4(1): 117-132.

Instituto Nacional de Estadística, Geografía e Informática (INEGI). 1988. Atlas nacional del medio físico. 224 pp.

Janzen, D.H. 1988. Tropical dry forests: The most endangered major tropical ecosystems. In: Wilson, E.O. (ed.), Biodiversity, pp. 130-137. National Academy Press, Washington, DC, USA.

Krebs, Ch. 1999. Ecological Methodology. Addison-Wesley. USA. 620 p.

Lima P.C.F., Lima, J.L.S. 1998. Composição florística e fitossociológica de uma área de Caatinga em Contendas do Sincorá, Bahia, Microregião. Acta Botanica Brasilica 12(3): 441-450.

López-Martínez, J.O., Sanaphre-Villanueva, L., Dupuy, J.M., Hernández-Stefanoni, J.L., Meave, J.A., Gallardo-Cruz,
J.A. 2013. $\beta$-Diversity of Functional Groups of Woody Plants in a Tropical Dry Forest in Yucatan. PLoS ONE 8(9): e73660. doi:10.1371/journal.pone.0073660.

López, O.R., Pérez, R., Mariscal, E. 2015. Diversidad de árboles y arbustos en fragmentos de bosque seco tropical en Rio Hato, Panamá. Colombia Forestal 18(1): 105-115.

López-Toledo, J.F., Valdez-Hernández, J.I., Pérez-Farrera, M.A., Cetina-Alcalá, V.M. 2012. Composición y estructura arbórea de un bosque tropical estacionalmente seco en la reserva de la biósfera la Sepultura, Chiapas. Revista Mexicana de Ciencias Forestales 3(12): 43-56.

Lott, E.J., Atkinson, T.H. 2010. Diversidad florística. En: Ceballos, G., Martínez, L., García, A., Espinoza, E., Bezaury, J., Dirzo, R. (eds.), Diversidad, amenazas y áreas prioritarias para la conservación de las selvas secas del Pacífico de México,pp. 63-76. Fondo de Cultura Económica, México.

Ludwig, J., Reynolds, J. 1988. Statistical Ecology. John Wiley \& Sons. New York, 337 pp.

Maass, M., Burgos, A. 2011. Water Dynamics at the Ecosystem Level in Seasonally Dry Tropical Forests. In: Dirzo, R., Mooney, H., Ceballos, G., Young, H. (eds.), Seasonally Dry Tropical Forests: Ecology and Conservation, pp. 141-156. Island Press. Washington. DC, USA.

Maass, M., Búrquez, A., Trejo, I., Valenzuela, D., González, M.A., Rodríguez, M., Árias, H. 2010. Amenazas. En: Ceballos, G., Benítez, L., García, A., Espinosa, E., Bezaury, J., Dirzo, R. (eds.), Diversidad amenazas y áreas para la conservación de las selvas secas del Pacífico de México, pp. 321-346. Fondo de Cultura Económica, México.

Magurran, A.E. 1988. Ecological diversity and its measurement. Princeton University Press, Princeton, 179 pp.

Magurran, A.E. 2004. Measuring biological diversity. Blackwell, Oxford. 256 pp.

Margalef, R. 1982. Ecología. Omega. España. 951 p.

Méndez-Toribio, M., Martínez-Cruz, J., Cortés-Flores, J., Rendón-Sandoval, F.J., Ibarra-Manríquez, G. 2014. Composición, estructura y diversidad de la comunidad arbórea del bosque tropical caducifolio en Tziritzícuaro, Depresión del Balsas, Michoacán, México. Revista Mexicana de Biodiversidad 85: 1117-1128.

Murphy, P.G., Lugo, A.E. 1986. Ecology tropical dry forest. Annual Review of Ecology and Systematics 17: 67-88.

Murphy, P.G., Lugo, A.E. 1995. Dry forests of Central America and the Caribbean. In: Bullock, S.H., Mooney, H.A., Medina, E. (eds.), Seasonally Dry Tropical Forests, pp. 9-34. Cambridge University Press, New York, USA.

Odum, E. 1972. Ecología. Interamericana. México. 639 pp.

Pennington, R.T., Lavin, M., Oliveira-Filho, A. 2009. Woody Plant Diversity, Evolution, and Ecology in the Tropics: Perspectives from Seasonally Dry Tropical Forests. 
Annual Review of Ecology, Evolution and Systematics 40: 37-57.

Pielou, E.C. 1975. Ecological Diversity. Wiley, New York. 165 pp.

Powers, J.S., Becknell, J.M., Irving, J., Pérez-Aviles, D. 2009. Diversity and structure of regenerating tropical dry forests in Costa Rica: Geographic patterns and environmental drivers. Forest Ecology and Management 258: 959-970

Riquetti, C.Ch., Soares, S.J.R., De Oliveira, D.A., De Mello, J.M., Mendonça, M.E.L. 2014. Diversidade e similaridade de fragmentos florestais nativos situados na região nordeste de Minas Gerais. Cerne 20(1): 1-10.

Rohwera S., Hobson, K., Rohwer, V.G. 2010. Reply to Urquhart: Conservation of Migratory Double Breeders. Proceeding of the National Academy of Sciences of the United States of America 107(5): E1 5.

Rubio, R.Y.G., Barcenas, H., Beltrán, A. 2010. Meseta de Cacaxtla, Sinaloa. En: Ceballos, G., Martínez, L., García, A., Espinoza, E., Bezaury, J., Dirzo, R. (eds.), Diversidad, amenazas y áreas prioritarias para la conservación de las selvas secas del Pacífico de México, pp. 405-410. Fondo de Cultura Económica, México.

Rzedowski, J. 1978. Vegetación de México. Limusa. México D.F., México. 432 pp.

Rzedowski, J., Calderón, G. 2013. Datos para la apreciación de la flora fanerogámica del bosque tropical caducifolio de México. Acta Botánica Mexicana 102:1-23.

Sánchez-Azofeifa, A. Calvo-Alvarado, J., Do Espírito-Santo, M.M., Fernandes, J.W., Powers, J.S., Quesada, M. 2014. Tropical dry forests in the americas: the tropidry endeavor. In: Sánchez-Azofeifa, A., Powers, J.S., Fernandes. G.W., Quesada, M. (eds.), tropical dry forests in the Americas ecology, conservation, and management, pp. 1-16. CRC Press, USA.

SAS Institute Inc. 2014. SAS/STAT13.2 user's guide: highperformance procedures. Cary, NC: SAS Institute Inc.
SEMARNAT Secretaría del Medio Ambiente y Recursos Naturales. 2010. Norma Oficial Mexicana NOM-059SEMARNAT-2010, Protección Ambiental-Especies Nativas de México de Flora y Fauna Silvestres-Categorías de Riesgo y Especificaciones para su Inclusión, Exclusión o Cambio-Lista de Especies en Riesgo. Diario Oficial de la Federación del 30 de diciembre de 2010.

SEMARNAT Secretaría del Medio Ambiente y Recursos Naturales. 2015. Resumen del plan de manejo del área de protección de flora y fauna Meseta de Cacaxtla. México D.F. 77 pp.

Sicairos, A.S., Díaz, J.S., Sánchez, G.S. 2003. Recursos bióticos silvestres en la zona costera de Sinaloa. En: Karam, C., Beraud, J.L. (eds.), Sinaloa y su Ambiente. Visiones del presente y perspectivas, pp. 281-328. Universidad Autónoma de Sinaloa, Sinaloa, México.

Smith, N.P., Mori, S.A., Henderson, H., Stevenson, D.W., Heald, S.V. 2004. Flowering plants of the Neotropics. The New York Botanical Garden. Princeton University Press. USA. $594 \mathrm{pp}$.

Trejo, I., Dirzo, R. 2002. Floristic diversity of mexican seasonally dry tropical forests. Biodiversity and Conservation 11: 2048-2063.

Towned, H.F. 1999. EcoStat for Windows. Win95/98/NT. Version 1.0.2. Loyola Marymount University. Trinity Software, Los Angeles, California. 77 pp.

Tropicos, Botanical information system at the missouri botanical garden. 2018. Búsqueda de nombres. hpttp://www. tropicos.org. Accedido febrero 23, 2018.

Vega, A., Bojórquez, B.G.A., Hernández, A.F. 1989. Flora de Sinaloa. SEP, UAS. 49 pp.

Vega, A. R., Villaseñor-Ríos, J.L. 2008. Listados Florísticos de Sinaloa. I. Flora del Municipio de Culiacán, México. UAS. $85 \mathrm{pp}$.

Williams-Linera, G., Lorea, F. 2009. Tree species diversity driven by environmental and anthropogenic factors in tropical dry forest fragments of central Veracruz, Mexico. Biodiversity and Conservation 18: 3269-3293.

Received: 12.02 .2018

Accepted: 15.05.2019 